\title{
The Relationship between Children's Developmental Functioning and Participation in Social Activities in Portuguese Inclusive Preschool Settings
}

\author{
Vera Coelho* and Ana Isabel Pinto \\ Faculty of Psychology and Educational Sciences, University of Porto, Porto, Portugal
}

In Portugal, substantial changes occurred in early childhood intervention and special education with the Decree-Law 3/2008 (2008). This legislation stresses the importance of a multidimensional approach to assessment-intervention processes, setting the International Classification of Functioning Disability and Health (ICF) as a critical tool in determining children's functioning and participation. To establish criteria for eligibility and

OPEN ACCESS

Edited by: Brahm Norwich, University of Exeter, United Kingdom

Reviewed by: Caroline Bond, University of Manchester, United Kingdom Gregor Ross Maxwell, UiT The Arctic University of Norway, Norway

*Correspondence: Vera Coelho

veracoelho@fpce.up.pt

Specialty section: This article was submitted to Special Educational Needs, a section of the journal Frontiers in Education

Received: 12 December 2017 Accepted: 15 February 2018 Published: 05 March 2018

Citation:

Coelho V and Pinto Al (2018) The Relationship between Children's Developmental Functioning and Participation in Social Activities in Portuguese Inclusive Preschool Settings. Front. Educ. 3:16. doi: 10.3389/feduc.2018.00016 defining the standard for developing assessment-intervention procedures in accordance with the ICF model, became topics of great relevance for researchers, practitioners, and policy-makers. Assuming that young children develop through proximal processes, such as playing and interacting with adults/peers/materials, their participation in everyday environments becomes a crucial outcome regarding learning and development, as highlighted by the ICF conceptual model. Children's participation can be defined as encompassing two dimensions: frequency of activities attended by children, and their level of engagement. This observational study analyzes engagement and time spent in activities with different levels of social complexity, in children with heterogeneous developmental functioning (DF). By focusing on different levels of DF, we aim to explore how functionality measures based on ICF can be useful to characterize all children, thus ensuring an inclusive assessment. Participants were 247 preschool-aged children: 54 with disabilities (low DF), 78 at-risk (medium-low DF); 115 with typical development (high DF). DF was assessed by a short-version of the Matrix for Assessment of Activities and Participation. Engagement and time in activities were observed with the Child Observation in Preschool. Analyses of Variance were conducted to explore differences between the three groups of children. The three DF groups differed significantly on the proportion of time spent in activities with different levels of social complexity. Children with disabilities spent less time in cooperative and social play (activities with higher social complexity) and their levels of engagement were significantly lower than the other groups. Discussion focuses on assessment demands posed by legislation and the ICF model, highlights the need to ensure all children's participation in different everyday activities, and underlines the role of environmental adjustments for children with disabilities and at risk to guarantee their right to fully participate in inclusive settings.

Keywords: participation, engagement, developmental functioning, inclusion, International Classification of Functioning Disability and Health - Children and Youth version, preschool 


\section{INTRODUCTION}

Children's right to participate in educational settings is a universal principle stated in several documents [e.g., UNESCO's Salamanca Statement and Framework for Action in Special Needs Education (UNESCO, 1994); UN General Assembly, 2007]. This right may be specially compromised in the case of young children with disabilities. Having that in mind, several countries have set as a major goal the development of measures and policies to ensure adequate supports and services that enable all children to fully participate in regular educational settings (Guralnick, 2008). In fact, participation outcomes have been considered by the World Health Organization (2013) as indicators of the quality of inclusion in services and policies. Children's participation in educational settings, as documented by the International Classification of Functioning Disability and Health - Children and Youth version (ICF-CY; World Health Organization, 2007), can constitute a way of monitoring the implementation of the United Nations Convention on Rights of People with Disabilities, article 24 regarding the educational settings' effectiveness in providing learning opportunities for all children (World Health Organization, 2013).

In Portugal, substantial changes occurred in Early Childhood Intervention (ECI) and in Special Education (SE) services with the implementation of the Decree-Law 3/2008 (2008). This legislation aims to support children's rights and stresses the importance of ensuring children's participation by approaching disabilities within a multidimensional perspective. This law aims to promote better quality support services in educational settings and defines the International Classification of Functioning Disability and Health (ICF; World Health Organization, 2001) as a crucial tool in the assessment process to document children's functioning and participation in educational settings as well as to determine adequate intervention measures in case of need for additional support. To achieve such purpose, developing assessment-intervention procedures for SE in accordance with the ICF model became a topic of great relevance for researchers, practitioners, and policy-makers, specifically in Portugal.

In Portugal, preschool-aged children, regardless of their characteristics, attend regular classrooms, meaning that preschools must be prepared to receive and adequately support all children, including those with disabilities. It is expected that preschool teachers plan activities in order to promote all children's participation, including those with disabilities or with any kind of developmental difficulties, by organizing the learning environment and interacting in a positive and responsive manner. Interventions with children with SE needs should occur in natural environments, meaning that the interactions teachers develop, and the activities they plan in the classroom, become main intervention targets (Gamelas, 2003; Pinto et al., 2012). The challenge for teachers is to adequately support children's specific developmental needs, while ensuring a high-quality responsive environment for the all group. Portuguese governmental guidelines for preschool education (Silva et al., 2016) refer the need to ensure a high-quality inclusive environment and underline the teacher's role in organizing the educational settings and planning activities to promote all children's development and participation.
Both structured activities and free-play are recommended to provide children's opportunities to explore the environment and to engage in individual, small-group and whole-group activities. However, a recent study has shown that Portuguese preschools are inconsistent in promoting diverse types of activities, suggesting the need for teachers to increase the amount of opportunities for children to participate in small-group and free-play activities (Pinto et al., 2014). Besides teachers' role in organizing the activities, the guidelines also highlight the need for teachers to develop positive interactions aiming to promote children's wellbeing and active role in their own learning, as well as to identify children's specific needs.

To discuss assessment procedures and eligibility criteria for SE based on children's functioning and participation in educational settings, we first need to define these constructs. Based on the tenets of the bioecological model (Bronfenbrenner and Morris, 2007) and on the ICF framework (World Health Organization, 2001), functioning comprises the way children use their individual and environmental resources, as well as their involvement in activities within natural settings (e.g., World Health Organization, 2001; Adolfsson et al., 2012; Norwich, 2016). Functioning is considered an umbrella term emerging from the bidirectional processes that occur between individuals' body functions, body structures, and environmental factors, resulting in activities and participation. In the ICF approach to functioning, the concept of participation is underlined and has been object of many debates among the scientific literature (e.g., Coster and Khetani, 2008; Granlund, 2013). Participation has been considered a health-related concept depicting transitional processes between the person and the environment (e.g., Eriksson and Granlund, 2004; Imms and Granlund, 2014; Imms et al., 2017). Although its definition is not consensual, authors agree that it is a multidimensional construct, portraying proximal processes (Simeonsson et al., 2001; Roper and Dunst, 2003; Almqvist, 2006; Dunst et al., 2006; Granlund, 2013). The WHO defines it as "involvement in life situations" (World Health Organization, 2001, p.18). In this line and in order to achieve a better understanding of the concept in educational settings, children's participation has been operationalized as encompassing two dimensions, namely the time spent in activities attended in natural settings and the level of engagement while attending such activities (Granlund, 2013; Imms and Granlund, 2014; Imms et al., 2017). Knowing that young children develop through proximal processes, such us playing and interacting with adults/peers/materials in their natural environments, both aspects of participation are found to be crucial for learning and development (Aydoğan et al., 2015). Considering this definition, literature emphasizes that children with disabilities often experience participation restrictions in its both dimensions, namely (1) attendance and (2) engagement in daily activities (e.g., Eriksson et al., 2007; Casey et al., 2012; Ferreira et al., 2012).

In terms of the attendance dimension, studies have shown that children with disabilities are more often excluded from activities when compared to their peers without disabilities and, therefore, need adequate interventions in order to improve participation outcomes (e.g., Eriksson et al., 2007). Considering the engagement dimension of participation, literature has shown that engagement with teachers, peers, and materials in daily activities 
in natural educational settings is associated with young children's development and learning (e.g., de Kruif and McWilliam, 1999; Pinto et al., 2006; Aydoğan, 2012). Based on several definitions provided in the scientific literature is it understood that children are engaged when they have an active role in tasks, activities, or routines (Odom and Bailey, 2001), and when they reveal appropriate interactions with their immediate physical and social environments (Bailey and Wolery, 1992; McWilliam and Bailey, 1992). According to McWilliam (2005) engagement refers to the time children spend in developmentally and contextually appropriate interactions with the physical and social environments, and covers the whole range of children's behaviors, providing a comprehensive picture of children's experiences in natural contexts.

This definition of engagement as encompassing developmentally and contextually appropriate interactions has raised some concerns in the literature focusing on engagement of children with disabilities within the ICF framework. In fact, how can engagement be studied in children whose behaviors and interactions might not become more complex over time or take longer to get more complex, due to effects of disabilities? Recently, two engagement dimensions were identified: a core dimension considered independent of chronological age, and a developmental dimension related to chronological age (Granlund, 2013; Sjöman et al., 2016). The authors argue that not all aspects of engagement are strictly related to a developmental hierarchy, such us problem-solving skills, or mastery motivation behaviors (Blasco et al., 1993; Malone et al., 1994; Sjöman et al., 2016) and, therefore, they can be interpreted as part of a core dimension of engagement. This core dimension of engagement is in line with Laevers' (Laevers, 1997, 2006) concept of involvement which is not related to specific behaviors or ages, but rather to a sense of wellbeing, flow and to feelings of belonging and comfort in the setting/activity (Coster et al., 2012; Granlund, 2013; Sjöman et al., 2016). For that, core engagement can be observed likewise in all children-babies, children with intellectual disabilities, and typically developing children-regardless of their developmental status (Sjöman et al., 2016). In literature and research on engagement in early childhood, some studies have focused on children's amount of time showing engagement (e.g., Wolery et al., 2000; Downer et al., 2007), while others measured its complexity (e.g., Kontos and Keyes, 1999; Raspa et al., 2001). Recently, Sjöman et al. (2016) reported findings that support this categorization of the engagement construct in two dimensions based on an exploratory factor analyzes in a study, including children with and without need of additional support. However, more research on these two dimensions of engagement is needed in order to better understand children's participation, regardless of their functioning, cognitive impairment, or developmental delay (Sjöman et al., 2016), particularly when analyzing children's participation in educational settings in light of the ICF perspective.

Based on the ICF perspective, in order to capture children's current functioning and participation (measured as time in activities and engagement) in educational settings, the use of norm-referenced tests is considered an inadequate approach. In fact the ICF model underlines the need for a multidimensional approach to assessment in natural environments (Simeonsson et al., 2003; World Health Organization, 2007; Bagnato et al.,
2010; Castro and Pinto, 2015), arguing that children's development must be contextualized and not assessed exclusively through abilities in standardized developmental tasks (Bagnato et al., 2010; de Sam Lazaro, 2017). The ICF framework stresses the need to go behind diagnosis and traditional developmental assessment categories and to portray transactional processes occurring between individuals and environments, by documenting each child's functioning and participation within a multidimensional perspective (education, health, and social care issues; World Health Organization, 2007). Thus, authentic-assessment measures and procedures are needed to conduct a comprehensive approach to children's functioning and participation (Majnemer, 2012; Castro and Pinto, 2013), in order to inform eligibility and intervention decisions in educational settings. This functionality approach to the assessment-intervention process is especially relevant in the case of children with atypical developmental trajectories or at risk. However, dynamic assessment procedures that may portray all children's performance in natural contexts are still lacking, in order to obtain a holistic and comprehensive understanding of children's actual participation and to plan adequate measures and ECI/SE services based on their needs, particularly in Portugal (Sanches-Ferreira et al., 2015; SilveiraMaia et al., 2017).

When aiming to analyze young children's functionality and participation in natural settings, play and exploration, interaction with peers, and appropriate learning challenges are relevant factors to consider for all children, with and without disabilities (World Health Organization, 2007; Brillante, 2017). Recognizing the relevance of play in child development the ICF includes, in its version for children and youth, a set of codes that allow documenting children's play (chapter 8: d880-engagement in play). Particularly social play, as an important component of the proximal processes, should be characterized by continuous, frequent and progressively more complex interactions in order to promote development (Bronfenbrenner, 1979). Play among peers in early ages can be characterized by interactions with increasing complexity in social demands (e.g., Rubin et al., 1976; Turnbull and Jenvey, 2006). Literature points out that young children move from less complex types of play, such as solitary play, to more complex, partner-dependent types of play, such as parallel play, associative play, or cooperative play (Rubin et al., 1976; Jamison et al., 2012). Typically developing children are able to progress from simple to more complex types of play without teacher special support. However, children with disabilities may experience restrictions in attending and in being engaged in activities requiring more complex levels of social play (Sigafoos et al., 1999), and may need adequate support from their environments to spend more time engaged in more complex social interactions with their peers. Literature shows that the social development of children with disabilities and their later social success can be greatly influenced by early interventions targeting social play between children with disabilities and their typically developing peers (Ivory and McCollum, 1999; Craig-Unkefer and Kaiser, 2002; Guralnick et al., 2006). Both peers and teachers, as key elements of children's social environments, can actively facilitate participation in all types of social play. In fact, beside children's characteristics, a diversity 
of environmental factors which can affect children's social play are highlighted in the literature, namely classroom characteristics (e.g., toys characteristics, the existence of learning/ activity centers), and the types of teacher interactions (Ivory and McCollum, 1999; Guralnick et al., 2006). As stated by the bioecological model of human development (Bronfenbrenner and Morris, 2007) and by the ICF approach to disability (World Health Organization, 2001), contextual factors may strongly facilitate the development of all children, but particularly the development of children with disabilities and/or at-risk as these children may experience restrictions in their participation in educational settings. Studies showed differences in the amount of time children with disabilities spent in various activity centers, in the number of different types of play in which they engaged, and in the amount of time they spent in different types of play, when compared to their typically developing peers (Brown and Bergen, 2002; Eriksson et al., 2007). This may indicate that the opportunities for children with disabilities and/or at risk to attending and participate in all types of activities in preschools are not being completely ensured.

The present study aims to contribute to operationalizing an approach to document children's functioning and dimensions of their participation in inclusive preschool settings based on the ICF framework. First, we explored how functionality measures based on the ICF-CY can be used to characterize all children in inclusive preschool settings. Then, we focused on analyzing dimensions of participation in children with heterogeneous DF characteristics regarding: (a) time spent in activities with different levels of social complexity and (b) engagement in activities with different levels of social complexity. The main question was: in inclusive preschool settings, how does individual time and engagement in activities with different levels of social complexity differ in children with different DF profiles?

\section{MATERIALS AND METHODS}

\section{Participants}

Participants were 247 preschool-aged children $(\mathrm{M}=52.75$; $\mathrm{SD}=6.83$ ) attending 42 classrooms from the district of Porto, Portugal. Following a random list of numbers, classrooms from the list of preschools identified at the ministry of education website for the referred district were contacted. Directors were contacted until 42 inclusive preschool classrooms agreed to participate. Overall, 80 Early Childhood Education (ECE) institutions were contacted, and 26 did not agree to participate. In addition, 11 were excluded once they did not meet the following criteria: being an inclusive classroom with at least one child eligible for ECI or SE support services attending. In each classroom, four to seven children were selected, including one child identified and receiving support from the ECI/SE services. The criteria for selection of the other children in the classroom were based on the teacher's assessment of children's developmental functioning (DF) level. Teachers completed a short-version of the Matrix for Assessment of Activities and Participation (MAAP; Castro and Pinto, 2012) for all children whose families had given consent to participate in the study. Overall, three groups were considered: (a) children with disabilities eligible for SE or ECI services; (b) at- risk children; and (c) typical developing children. As mentioned, for the group of children with disabilities, children were automatically selected as they were previously identified as eligible for SE or ECI. At least one child with disabilities was selected in each classroom; in 12 classrooms two children with disabilities were selected. For the groups, children at-risk and children with typical development, the MAAP scores were used to select for each classroom: two children with the lowest scores in the MAAP, for the group at-risk; and three children randomly selected among the children with the highest scores in the MAAP, for the typically developing group. This selection allowed capturing the diversity of DF characteristics in children attending each classroom.

Overall, participants included 54 children eligible for SE/ECI support due to identified disabilities; 78 children considered at-risk; and 115 typically developing children. Diagnostic categories documented for the group of children with disabilities were diverse: 20 children were identified with Global Developmental Delay; 17 children were identified with Autism Spectrum Disorders; four children were identified with Cerebral Palsy; three children were identified with Down Syndrome; two children were identified with Language Delays; two children were identified with Hyperactivity; 1 child was identified with a Cardiac Condition; one child was identified with Hearing Deficit; and four children were identified with rare syndromes, such as Kabuki Syndrome, Costello Syndrome, and Cri-du-chat Syndrome. The three groups of children - with disabilities, at-risk and typically developing-had similar ages, $F(2,234)=38.89$, $p=0.44(\mathrm{M}=53.34, \mathrm{SD}=7.53 ; \mathrm{M}=51.92, \mathrm{SD}=6.57$; and $\mathrm{M}=52.00, \mathrm{SD}=6.65$, respectively). However, and similarly to previous studies (e.g., Lai et al., 2012; Grande, 2013) the group of children with disabilities included a much higher number of boys $(82 \%$ in the group children with disabilities; $65 \%$ in the group children at risk; $52 \%$ in the group typically developing children).

Classrooms had on average 20.98 children ( $S D=2.98$ ), including at least one child with disabilities eligible for SE or ECI. The number of children with disabilities per classroom varied between 1 and $5(\mathrm{M}=1.63, \mathrm{SD}=0.83)$. Teachers were all female with ages between 27 and 59 years old $(M=50.04, S D=6.58)$. All teachers had, at least, a degree in preschool teaching, with an average of 16.09 years of formal education $(\mathrm{SD}=0.42)$. Their experience as a preschool teacher varied between 2 and 36 years $(M=25.50$, $\mathrm{SD}=7.13$ ). Similarly, teachers' experience in inclusive classrooms ranged between 1 and 30 years $(M=11.66, S D=8.57)$.

The Portuguese National Data Protection Authority and the Committee for Monitoring Studies in Education Settings of the General Direction of the Ministry of Education approved all measures and data collection procedures (authorization no. $16785 / 2015$ and authorization no. 0535000001, respectively); informed consent was obtained from the preschool directors, preschool teachers and families. No additional ethical authorizations were required. The mentioned approvals analyzed all ethical issues regarding the study. Written informed consent was obtained from the preschool directors, preschool teachers, and families. 


\section{Measures}

Matrix of Assessment of Activities and Participation (MAAP; Castro and Pinto, 2012)

The MAAP measure was used to assess children's DF. This is a measure designed for children between 2 and 6 years old and assesses functionality in accordance to the World Health Organization (2007) guidelines for ECI assessment-intervention processes. It was developed using a Delphi Method and based on the linkage between commonly used developmental measures (e.g., Griffiths, Schedule of Growing Skills) and the ICF-CY codes (Castro, 2012; Castro and Pinto, 2015). MAAP items were created to portray the contents of developmental measures corresponding to the ICF-CY codes and provide a functionality profile including information on child body functions, activity and participation, and environmental factors. Although there are not many studies on the MAAP, the measure have shown to be adequate in differentiating children's functioning, namely between children with disabilities and typically developing children (Castro and Pinto, 2015), and with at-risk populations (Caetano, 2014). It consists of a questionnaire that can be completed by teachers or other professionals that regularly interact with the child in his/ her natural environments. Each item is scored in a scale ranging from 1 (the child has a total difficulty in the domain described) to 5 (the child is totally autonomous in the domain described). An overall score is obtained by averaging the scores of all items: higher scores represent higher levels of functionality of the child in preschool context. Authors report good reliability values of the MAAP (Cronbach's alpha varied between 0.76 and 0.98 ) and significant differences, in several dimensions of the measure, between the scores of children with disabilities and typical developing children (Castro and Pinto, 2015).

The short-version of the MAAP used in the present study was composed by six items, selected based on the ICF-CY Developmental Code Sets. The Developmental Code Sets were developed through the Delphi Method, considered by experts to be relevant for the DF of children in the age groups 0-2 and 3-5 (Ellingsen, 2011). The MAAP short-version included the following items: basic knowledge skills, related to the ICF-CY code d137; ability to perform a single task, related to the ICF-CY code $\mathrm{d} 210$; involvement in preschool tasks, related to the ICF-CY code d815; conversation, related to the ICF-CY code d330; attention, related to the ICF-CY code b140; and play competences, related to the ICF-CY code d880. Cronbach's alpha for the short-version of the MAAP used in this study was 0.94 , similarly to previously reported value (Guichard and Grande, 2017).

In this study, the option to use this authentic-assessment measure relies on the fact the most recent approaches in ECE and care including the ICF-CY approach, highlight the importance of functioning in daily contexts for assessment-intervention processes in SE or ECI (e.g., Simeonsson et al., 2003; World Health Organization, 2007; Bagnato et al., 2010; Majnemer, 2012; de Sam Lazaro, 2017); In addition, several authors underline that traditional norm-reference developmental assessments are inadequate for children whose development is not following the normative path, highlighting that such norm-referenced assessments tend not to be sensitive to children disability characteristics (Bagnato, 2007; Macy et al., 2015). Based on these assets, and on the ICF framework we considered that using a norm-reference development assessment would not allow to capture differences in children's functioning in preschool, regardless of their diagnosis. Lastly, and to our knowledge, the MAAP is unique as a measure that documents children's DF based on the ICF-CY, an approach that is recommended by Portuguese legislation for teachers to identify and assess children at-risk/with disabilities.

\section{Child Observation in Preschool (COP; Farran and}

\section{Anthony, 2014)}

The COP measure was used to assess children's engagement. This measure consists of an observation system that captures children's behavior in preschool settings, throughout a typical day. It uses a systematic behavior-sampling procedure, known as a "snapshot" procedure, to collect information on: (a) children's listening and (b) verbal behaviors, (c) learning setting-schedule, (d) proximity to and (e) interaction state, (f) activity and tasks demands, (g) materials and (h) focus of activities, and (i) level of involvement. Each child is observed for $3 \mathrm{~s}$, after which the observer immediately codes all categories. Children must be observed over 20 snapshots, or sweeps, across the preschool day. COP has been used in several studies, with different samples, that report its validity (e.g., Lillvist, 2010; Luttropp and Granlund, 2010; Fuhs et al., 2013; Nesbitt et al., 2015).

Similarly to other studies, in which COP dimensions are used independently or combined (e.g., Fuhs et al., 2013; Nesbitt et al., 2015), the present study analyzes data from two of the COP dimensions-Involvement and Interaction State. Involvement is coded in a 5-point scale, where 1 means low engagement (e.g., totally out of task, not paying attention to the activity, sitting quietly; fiddling with another child's hair or clothing, eyes not focused on ongoing activity); 2 means medium-low engagement (e.g., looking at teacher and/or material inconsistently, flat affect, looking bored, visible attention going in and out, visible lack of persistence); 3 means medium engagement (e.g., on task, maintaining eye contact with teacher, participating but may briefly look around but immediately comes back to task); 4 means medium-high engagement (e.g., eager expression, relevant self-talk during tasks, volunteering response with positive affect, looking at material throughout entire time; leaning forward, showing persistence); and 5 means high engagement (e.g., intense focus, serious persistence and pursuit of activity, very difficult to be distracted from the activity, seeming oblivious to noise and the behaviors of the other children that are not related to the task). This dimension score is computed by averaging the values of all observation sweeps for each child. For computing level of engagement in each type of social play, involvement scores must be averaged by each type of play coded in the Interaction State dimension (e.g., average of all involvement scores for all sweeps coded as cooperative play for each child to find the score for engagement in cooperative play).

Interaction State is a categorical dimension related to the social parameters of the child's learning and development experiences in preschool. This dimension assumes that children's interactions can be partially determined by child's preferences, child's developmental competence, and by the environment organization, i.e., by what teacher allows and plans. Coding options in 
this dimension are based on Parten's (1932, cit in Rubin et al., 1976; Farran and Anthony, 2014) widely used description of different types of social play activities, including unoccupied (i.e., the child is not doing anything); solitary play (i.e., the child plays alone in proximity to others or not, using unique materials and not interacting with others); onlooker (i.e., the child observes an activity in which is not participating); parallel play (i.e., the child uses similar materials, is doing similar activities as peers, but they are working independently), associative play (i.e., the child is sharing materials and interacting, but no distinguishable goal for the group is visible); and cooperative play (i.e., the child works together with peers/teachers, having shared goals, rules, and/or organization). The Interaction State category also includes a code for classroom routines like hand-washing, toileting, or waiting on the rug, denominated non-academic, and a code for time-out moments. In summary, Interaction State is organized in a hierarchy of complexity regarding social play activities, ranging from unoccupied to cooperative play. Considering Parten's theory-still considered to be one of the most comprehensive descriptions of children's social play (Rubin et al., 1976; Xu, 2010)_unoccupied, onlooker behavior and solitary play can be considered non-social activities; parallel play is considered a limited form of social play; and associative and cooperative play are considered true social play. This dimension score is determined by the proportion of sweeps observed in each category during the observation period. For instance, by computing the number of sweeps observed for a particular child in cooperative play/total of sweeps observed for that child, we obtain the proportion of time the child spends in cooperative play. Non-academic and time-out codes are not included in this hierarchy for complexity of social play and, for that, were also not analyzed in the present study.

Researchers collecting data received training on the COP measure, including theoretical sessions, video coding and discussion, and in-context observation for inter-observer agreement reliability. For reliability purposes, inter-observer agreement was checked for $25.05 \%$ of children observed during data collection. Overall, exact inter-observer agreement varied between 80.07 and $98.42 \%$, with a global average of $95.73 \%$. Specifically, the involvement dimension had an exact inter-observer agreement of $80.07 \%$; Agreement within one point of difference was 98.46\%; weighted kappa was 0.78 , showing good reliability; Interaction State dimension reached $97.5 \%$ of exact inter-observer exact agreement.

\section{Procedures \\ Data Collection}

Data were collected between January and May 2016 in two moments. First, the MAAP measure was completed based on an individual interview format with the teachers. This first assessment intended to select the target children in each classroom. Second, an observation moment in the preschool classroom was conducted. The COP measure was completed within a $3 \mathrm{~h}$ observation in the classroom during a typical preschool morning. On average, whole-group activities were observed for $47 \%$ of the time, free-play for $12 \%$, small-group activities for $0.3 \%$, free-play and small-group activities (at the same time), mealtime, transitions, and playground were observed, each, for approximately $10 \%$ of the time.

\section{Data Analyses}

To examine the extent to which the three groups of children differ in time spent in activities with different levels of social complexity analyses of variance (ANOVA) were computed. The group of children with disabilities included children with different diagnostic categories which were not considered in the analyses. The same procedure was used to compare the three groups of children in the overall levels of engagement and in the levels of engagement by type of activity with different levels of social complexity. Effect sizes were estimated using the partial eta squared $\left(\eta_{\mathrm{p}}^{2}\right)$ and interpreted according to Cohen's guidelines (Cohen, 1988): $\eta_{\mathrm{p}}^{2}=0.01$ indicates a small effect; $\eta_{\mathrm{p}}^{2}=0.06$ indicates a medium effect size; and $\eta_{\mathrm{p}}^{2}=0.14$ indicates a large effect size.

\section{RESULTS}

\section{Functional Perspective in Assessment}

Results regarding the assessment of all children in inclusive educational settings based on a functionality approach, showed significant differences in the MAAP short-version global score, $F(2,243)=226.194, p<0.001, \eta_{\mathrm{p}}^{2}=0.65$, between the three groups of children included in this study: with disabilities, atrisk and with typical development. Post hoc analyses, specifically Tukey HSD showed that all pairs were significantly different in the MAAP short-version global scores $(p<0.001)$ with the group of children with disabilities presenting the lowest scores regarding functionality characteristics (see Table 1). Similarly, significant differences between the three groups were also found for all items included in the MAAP short-version, when these are analyzed individually (see Table 1). Effect sizes were large for all differences found between the groups.

\section{Participation in Preschool: Time Spent in Social Play Activities and Levels of Engagement}

Results from the COP showed that, on average, all children in the three groups - with disabilities, at risk and typically developingspent the majority of their time in parallel play activities. Also for the three groups of children, the less frequent social play activity observed was the cooperative play. When analyzing each group according to the proportion of time spent in each activity, we can see that for children with disabilities the second most coded category was unoccupied (15\% of observed time), followed by onlooker behaviors (14\%) and playing alone (13\%). Associative play, for this group of children, was observed less than $7 \%$ of the time, social activities were observed during $4 \%$ of time and cooperative play was observed for $2 \%$ of time. For the group of children at-risk, the second most coded category was onlooker behaviors, followed by the associative play and unoccupied time (both observed for $11 \%$ of time each). Social play activities were observed during $7 \%$ of time for children at risk, playing alone for $4.1 \%$ of time, and cooperative play for $3.6 \%$ of time. For the typically developing group of children, the second most coded category was onlooker behaviors, followed by associative play ( $12 \%$ of time). These children spent $7.6 \%$ of the time unoccupied, $7.2 \%$ in social play, and $4.1 \%$ in cooperative play. 
TABLE 1 | Mean differences for each of the MAAP-short version measure item/International Classification of Functioning Disability and Health - Children and Youth version (ICF-CY) code, between the three groups of children: with disabilities, at-risk and typically developing.

\begin{tabular}{|c|c|c|c|c|c|}
\hline \multirow[t]{2}{*}{$\begin{array}{l}\text { MAAP item/ICF-CY } \\
\text { correspondent item }\end{array}$} & $\begin{array}{l}\text { With disabilities [low developmental } \\
\text { functioning (DF)] }\end{array}$ & $\begin{array}{c}\text { At-risk } \\
\text { (medium DF) }\end{array}$ & $\begin{array}{l}\text { Typically developing } \\
\text { (high DF) }\end{array}$ & $\boldsymbol{F}$ & $\eta_{p}^{2}$ \\
\hline & M (SD) & M (SD) & M (SD) & & \\
\hline Global score & $2.83(1.07)^{\mathrm{a}}$ & $3.58(0.47)^{\mathrm{a}}$ & $4.79(0.26)^{a}$ & $226.19^{\star \star \star}$ & 0.65 \\
\hline Basic knowledge skills [d137] & $2.78(1.36)^{\mathrm{a}}$ & $3.23(0.88)^{\mathrm{a}}$ & $4.71(0.53)^{\mathrm{a}}$ & $113.45^{\star \star \star}$ & 0.48 \\
\hline Ability to perform a single task [d210] & $2.72(1.31)^{\mathrm{a}}$ & $3.26(0.76)^{\mathrm{a}}$ & $4.75(0.49)^{\mathrm{a}}$ & $139.48^{\star \star \star}$ & 0.53 \\
\hline Conversation [d330] & $2.54(1.30)^{\mathrm{a}}$ & $3.64(0.90)^{a}$ & $4.80(0.44)^{\mathrm{a}}$ & $139.63^{\star \star \star}$ & 0.54 \\
\hline Play competences [d880] & $3.30(1.25)^{\mathrm{a}}$ & $4.22(0.75)^{\mathrm{a}}$ & $4.90(0.30)^{\mathrm{a}}$ & $85.61^{\star \star \star}$ & 0.41 \\
\hline Involvement in preschool [d815] & $3.22(1.18)^{\mathrm{a}}$ & $4.08(0.82)^{\mathrm{a}}$ & $4.92(0.30)^{\mathrm{a}}$ & $99.32^{\star \star \star}$ & 0.45 \\
\hline Attention skills [b1400] & $2.41(1.02)^{\mathrm{a}}$ & $3.08(0.79)^{\mathrm{a}}$ & $4.65(0.61)^{\mathrm{a}}$ & $187.27^{\star \star \star}$ & 0.61 \\
\hline
\end{tabular}

${ }^{* * *} p<0.001$.

Groups with equal letters present significant differences.

TABLE 2 | Mean differences between the proportion of time spent and engagement levels in activities with different levels of social complexity, between the three groups of children: with disabilities, at-risk, and typically developing.

\begin{tabular}{|c|c|c|c|c|c|c|c|c|}
\hline & \multicolumn{2}{|c|}{ With disabilities } & \multicolumn{2}{|c|}{ At-risk } & \multicolumn{2}{|c|}{ Typically developing } & \multirow[t]{2}{*}{$\boldsymbol{F}$} & \multirow[t]{2}{*}{$\eta_{p}^{2}$} \\
\hline & $N$ & M (SD) & $n$ & M (SD) & $n$ & M (SD) & & \\
\hline \multicolumn{9}{|l|}{ Time in } \\
\hline Cooperative play & 54 & $0.016(0.038)^{\mathrm{a}}$ & 78 & $0.036(0.057)$ & 115 & $0.041(0.059)^{a}$ & $4.02^{\star}$ & 0.032 \\
\hline Social play & 54 & $0.038(0.052)^{a, b}$ & 78 & $0.069(0.073)^{b}$ & 115 & $0.072(0.069)^{a}$ & $5.29^{\star \star}$ & 0.042 \\
\hline Associative play & 54 & $0.069(0.081)^{a, b}$ & 78 & $0.110(0.114)^{b}$ & 115 & $0.120(0.010)^{a}$ & $4.96^{\star \star}$ & 0.039 \\
\hline Parallel play & 54 & $0.269\left(0.163^{a, b}\right.$ & 78 & $0.343(0.151)^{b}$ & 115 & $0.357\left(0.142^{a}\right.$ & $6.70^{\star \star \star}$ & 0.052 \\
\hline Onlooker & 54 & 0.135 (0.084) & 78 & $0.133(0.094)$ & 115 & $0.129(0.082)$ & 0.11 & \\
\hline Alone & 54 & $0.127(0.137)^{a, b}$ & 78 & $0.041(0.049)^{b}$ & 115 & $0.049(0.060)^{\mathrm{a}}$ & $21.60^{\star \star \star}$ & 0.150 \\
\hline Unoccupied & 54 & $0.153(0.157)^{\mathrm{a}}$ & 78 & $0.110(0.110)$ & 115 & $0.076(0.082)^{a}$ & $9.65^{\star \star \star}$ & 0.073 \\
\hline \multicolumn{9}{|l|}{ Engagement in } \\
\hline Overall activities & 54 & $2.15(0.47)^{\mathrm{a}}$ & 78 & $2.90(0.37)$ & 115 & $2.90(0.38)^{\mathrm{a}}$ & $5.44^{\star}$ & 0.043 \\
\hline Cooperative play & 8 & $3.02(0.58)$ & 27 & $2.97(0.41)^{\mathrm{a}}$ & 41 & $3.39(0.62)^{\mathrm{a}}$ & $5.02^{*}$ & 0.120 \\
\hline Social play & 20 & $3.13(0.39)$ & 45 & $3.18(0.50)$ & 72 & $3.06(0.31)$ & 1.21 & 0.018 \\
\hline Associative play & 30 & $3.40(0.47)$ & 53 & $3.47(0.48)$ & 86 & $3.35(0.48)$ & 0.98 & 0.012 \\
\hline Parallel play & 51 & $3.05(0.58)$ & 73 & $3.22(0.47)$ & 109 & $3.22(0.39)$ & 2.08 & 0.018 \\
\hline Onlooker & 49 & $2.65(0.58)$ & 69 & $2.67(0.48)$ & 103 & $2.64(0.57)$ & 0.13 & 0.001 \\
\hline Alone & 39 & 3. $46(0.59)$ & 32 & $3.47(0.72)$ & 57 & $3.51(0.64)$ & 0.91 & 0.002 \\
\hline
\end{tabular}

${ }^{*} p<0.05,{ }^{* *} p<0.01,{ }^{* * *} p<0.001$.

Analyzes on levels of engagement were not computed to the unoccupied category as this implies that the child is not doing any activity/not playing and, consequently, low engagement levels are scored when this category is selected.

Groups with equal letters present significant differences.

When analyzing differences among the three groups, results showed that groups differ significantly in the time spent in activities with different levels of social complexity (see Table 2). Specifically, children with disabilities spent less time in activities with higher social demands such as cooperative, social, associative, and parallel play, and were observed spending significantly more time playing alone or unoccupied, when compared with the typically developing group. The group of children at risk also spent significantly more time in social, associative, parallel play when compared to the group of children with disabilities. No significant differences were found between the group children at risk and the group of typically developing children regarding time spent in all the observed activities. No significant differences between the three groups were found in time spent in onlooker behavior while playing.

Results from the engagement analyzes showed that the three groups of children presented, on average, low to medium engagement levels in all social play activities observed. Overall engagement for children with disabilities was low $(M=2.15$, $\mathrm{SD}=0.47)$, and similar average values were found when these children are engaged in onlooker behaviors with peers $(\mathrm{M}=2.65$, $\mathrm{SD}=0.58$ ). The remaining social play activities observed registered levels of engagement near the medium point of the scale. For the group of children with disabilities alone play and associate play received the higher engagement average scores. Both at-risk children and typically developing children presented medium overall engagement levels (see Table 2). For the group of children at risk, associative play registered the highest engagement levels and onlooker behaviors registered the lowest scores. The group of typically developing children presented the highest average engagement levels during alone play and cooperative play, while its lowest scores were registered for the onlooker behaviors.

Significant differences in overall engagement levels were found between children with disabilities and typically developing 
children, indicating that the group of children with disabilities presents lower engagement levels when compared with the group of children with typical development. No differences were found between overall engagement when comparing the at-risk group of children with the two other groups. When analyzing engagement by type of play activities with different levels of social complexity, results showed a significant difference between children at-risk and typically developing children in cooperative play indicating that at-risk children show lower engagement in this type of play. Regarding children with disabilities, only eight children were observed in cooperative play, thus, not allowing for analyzes of engagement differences with the other groups for this type of play (see Table 2). No significant differences between the three groups of children were found in the remaining types of social play activities observed in the present study.

\section{DISCUSSION}

This study aimed to contribute to operationalizing an approach to the assessment of child functioning in inclusive preschool settings based on the ICF framework, and to analyze dimensions of children's participation in preschool activities. First, we explored how functionality measures based on the ICF-CY can be used to characterize all children in inclusive preschool settings. Then we analyzed dimensions of participation in children with heterogeneous DF regarding: (a) time spent in activities with different levels of social complexity and (b) engagement levels in activities with different levels of social complexity.

Since the publication of the ICF, its application in educational contexts has raised several challenges in researchers and professionals' practices. Challenges regarding assessment are among the most relevant. In order to be in accordance with the ICF model, the assessment process must be able to capture DF aspects of all children, at different levels-namely body functions and structures, participation, and environmental variables. Murphy (2006) underlines that children's assessment is among the hardest tasks to solve in educational settings, and frequently leads to several debates. Traditional diagnostic assessments do not meet the most recent and relevant recommendations for preschool inclusive and equalitarian assessment (Lebeer et al., 2012) and thus, the use of the ICF framework provides a model for a comprehensive understanding of the child and its development as it highlights the relevance of transactions between body functions and structures, activities and participation, and environmental factors, regardless of diagnosis categories (Campbell and Skarakis-Doyle, 2007; Norwich, 2016). Regardless the recognition of its relevance, conceptually and by the SE legislation, environmental factors were found to be the less frequently assessed and targeted for intervention by Portuguese EI/SE professionals (Castro et al., 2014). Considering that the present study did not document environmental factors, it would be relevant to expand this research to include all ICF domains that may relate to children's participation. By accounting for physical and social characteristics of preschool environments, we would be in a better position to inform practitioners about the transactional processes that may contribute children's participation.
In Portugal, studies on the implementation of the ICF model on the assessment-intervention processes in inclusive educational settings (in accordance with the Decree-Law 3/2008, 2008) have shown that educational contexts are still failing to provide an integrated and dynamic view of children's functioning that adequately supports the definition of intervention strategies. There is a lack of assessment procedures based on a functional approach that are able to depict children's capacity and their performance levels with reference to environmental factors (Sanches-Ferreira et al., 2015).

Therefore, it is relevant to develop and study measures that enable an integrated and dynamic characterization of every child's functioning, enabling the design of adequate supports in inclusive settings. This is the case of the measure used in the present study - the MAAP-a measure based on the ICF-CY, which assesses the functionality of all preschool children. A short version of the MAAP was used, including a limited number of items related to body functions, activities, and participation components. However, although no items related to environmental factors were included in this version, our results show that this assessment tool allowed the identification and differentiation of children's functioning in preschool in an accurate way, independently of their diagnosis. Differences in functioning were captured, not only between the groups of children usually identified by preschools-typically developing children and children with disabilities/receiving support-but a third group of children was identified. This third group-group of "at-risk" children-shared some functioning and participation characteristics with children with disabilities and other with typically developing children. Despite eligibility criteria for ECI (DecreeLaw 281/2009) services in Portugal includes risk factors, children identified "at risk" in this study were not receiving any formal additional support. Nevertheless, teachers identify limitations in their daily functioning, and results from the present study show that their participation characteristics have similarities with both high and low functioning children. As such, although the ICF model is set by the legislation to frame practices in SE/ECI, the assessment of risk factors to be eligible for ECI in Portugal seldom includes difficulties in children's functioning, rather focusing on other criteria commonly referred in the literature (e.g., parent mental health conditions, poverty, parents low level of education, parents' unemployment, and adolescent maternity). In light of the ICF model, it would be important to articulate both types of criteria when evaluating children's need for support in preschool inclusive settings. This does not mean that all children identified in our study as being "at risk" should be eligible for ECI. But we believe that all children in education settings should be able, at any point of their educational trajectory, to be eligible to receive adequate support, similarly to the multi-tiered systems of support model (Grisham-Brown and Pretti-Frontczak, 2011; McConnell et al., 2014).

Considering that the Portuguese legislation (Decree-Law $3 / 2008,2008$ ) states that children are in need of special support when their participation in educational settings is restricted, and sets the ICF as a crucial tool in determining children's functioning and participation, this study analyzed participation in inclusive preschool settings, thus contributing to characterize aspects 
of inclusion in Portugal. Regarding children's participation in preschools, our results are in line with other studies which found that children with disabilities tend to be more excluded from preschool activities and to spend more time non-engaged or engaged in activities with lower complexity, when compared with their typically developing peers (e.g., Eriksson et al., 2007; Casey et al., 2012; Grande, 2013). Particularly the present study found that children with disabilities spent significantly more time in less complex social play, such as playing alone or even unoccupied, and presented lower levels of overall engagement than their typically developing peers. However, it is important to state that when analyzing engagement by type of social play, no differences in engagement levels were found between the three groups for the types of social play with lower or medium levels of complexity. This might be due, in part, to the fact that we focused on children's core engagement which is, according to Laevers' (Laevers, 1997, 2006) and others (e.g., Coster et al., 2012; Sjöman et al., 2016), not related to chronological age but to a sense of wellbeing and belonging. This particular result of the present study seems to support the idea that, with adequate support, similar levels of core engagement can be observed likewise in all children, regardless of their developmental status (Sjöman et al., 2016). In addition, as all children in preschool exhibit mediocre levels of engagement, we may hypothesize that the environments are not supporting children's engagement, regardless of their disability conditions. Supporting this hypothesis, several studies in Portugal have reported mediocre levels of quality of preschool environments (e.g., Cryer et al., 1999; Abreu-Lima et al., 2013; Pinto et al., 2014), and the need to increase quality in Portuguese inclusive classrooms (e.g., Aguiar et al., 2010; Pinto et al., 2014). Although it was not our goal in the present study, it would be of interest to analyze children's participation in relation to ecological factors such as availability of materials, classroom setting organization (e.g., whole-group, free-play) while accounting for different interaction partners (e.g., teachers, peers). In light of the ICF model, research including children as active informants about their own participation, their preferences and perceptions of the environment, would also add to a more comprehensive understanding of participation processes in preschools.

Furthermore, knowing that children with disabilities often need adequate support to be able to fully participate in their natural environments, it is not surprising that this group of children is not able to spend more time in more complex levels of social play. As all participation occurs within a specific setting and is affected by the contextual characteristics of that setting, such lack of adequate support is classified by the ICF as an environmental barrier to children's participation. As such, our results point to the possible gap existing between the legislation, which sets a multidimensional approach considering transactions among body functions, activities, participation, and environmental factors, and the assessment-intervention processes implemented in educational settings that still lack in adequately support all children's participation. Developing functional assessments that focus not only on children's characteristics but also on environmental aspects are in line with the concept of development as the result of transactions between individuals and environments. As such, the ICF model can constitute a valuable resource in promoting preschool environments that effectively support all children's development and participation, as it enables the analyses of each child's specific environments, providing guidelines for key environmental aspects that must be assessed and altered in order to reduce barriers and increase facilitators, thus optimizing the quality of inclusion and learning. Such guidelines would be of great value for designing teachers' initial training and to inform the development of policies that ensure children's right to fully access and participate in educational environments.

Moreover, our study found that, although the group of children at-risk presents a pattern of attendance of social play activities similar to typically developing children, when we analyze engagement levels in activities with higher levels of social complexity, children at risk show significantly lower patterns of engagement in more complex social play when compared to their typically developing peers. These children assessed to be at risk based on a functional measure, showed similar levels of engagement to the ones exhibited by children with disabilities who were considered eligible for ECI/SE services. This result indicates that a functional assessment may enable the identification of children who have a higher risk of presenting lower participation in their settings and that would not, otherwise, be effectively identified and consequently supported in preschools. Although the need to implement procedures for the early identification of children that may be at-risk has been stated in the literature (e.g., Luthar et al., 2015), to our knowledge, research on risk has focused mainly on biological and/or environmental risk factors and not on a functionality approach to children's problems as early indicators of developmental risk. An ECE model focusing on children's functionality, and accounting for transactional processes between body functions and structures, environmental factors, and activities and participation is needed in order to plan adequate supports according the Portuguese legislation framework.

Globally, the present study's results underline the need for assessments within a functional approach in order to mobilize adequate supports for children's participation and development and to support all agents in educational contexts. This study focused particularly on participation in preschool social play activities as several studies show that children in need of special support have difficulties related to social relations (e.g., Odom et al., 1992; Semrud-Clikeman, 2007; Lillvist, 2010). Given that children develop and learn in interaction with their proximal physical and social environments, there is a strong conceptual justification for designing classroom environments that promotes children's active engagement in diverse social activities. Play and exploration, interaction with peers, and appropriate learning challenges are important for all children, and children with disability or developmental delays should have the same opportunities to engage in such learning opportunities as their typically developing peers (Brillante, 2017).

Some limitations to this study must be acknowledged. First, we are aware that the assessment conducted with the MAAP shortversion does not capture all mandatory aspects of children's functioning according to the ICF model. No environmental factors were considered in the short-version of the measure and a limited number of items related to body functions, activities, and participation dimensions were included. The MAAP short-version 
revealed, however, to be an adequate tool for our purposes, namely to describe all children's functionality and to distinguish different functionality profiles in inclusive classrooms. Future studies should use the original complete version of the MAAP in order to provide a broader, multidimensional, dynamic and comprehensive assessment of child's DF. More research is also needed on the MAAP validity and reliability, namely regarding teachers' scoring reliability. Second, although this study included 247 preschool children, the number of children in each group was very discrepant and gender parity was not achieved. It would be important to conduct studies involving a higher number of classrooms so that the group of children with disabilities could be increased. Moreover, children in the at-risk group presented a wider range of functional characteristics probably due to the selection procedure. This might be due to the fact that each classroom's own diversity was considered as part of the selection criteria, once the two children with the lowest scores in the MAAP short-version measure were selected for the at-risk group. As such, the option for an approach with cut-off points for the MAAP short-version measure during the selection procedure could contribute to a more homogenous group of children for the at-risk group, which could lead to more consistent results and stronger effect sizes. Nevertheless, we recognize, as others (e.g., McManus et al., 2014) that there is notable heterogeneity in characteristics of vulnerable children and more research is needed particularly using a functionality approach to characterize at-risk children. Lastly, our data were restricted to one district of Portugal; and observations were conducted during one morning, thus confining the engagement data on less frequent types of social play and restricting analyzes regarding differences in engagement in more complex social activities.

\section{CONCLUSION}

Overall, the present study showed that the use of a functionality measure based on the ICF-CY in inclusive classrooms can contribute to document important DF characteristic for all children. Such assessment was found to be able to differentiate children, showing that at-risk children, children with disabilities, and typically developing children present significant differences in their functionality in preschool. This paper also intended to analyze two dimensions of participation, namely time spent in activities with different levels of social complexity and engagement levels in such activities, in these three groups of children. We found that, although typically developing children and children at-risk showed a similar pattern of attendance in social play activities, the engagement levels presented by at-risk children were significantly lower in activities with higher levels of social complexity compared to their typically developing peers. At-risk children showed similar levels of engagement to the ones presented by children with disabilities in more social complex activities.

Results emphasize the contribution of using a functional approach in assessment in inclusive settings to document all children's needs and to ensure their participation. Results also contribute to highlight that children's universal right to participate in educational settings may not always be consistently ensured in Portugal. Participation in natural environments, including time spent in activities and engagement, is crucial for every child learning and development. No children, including those with disabilities or with developmental delays should be deprived of experiences or learning opportunities in their natural environments (Brillante, 2017). Inclusion requires that every individual child receives adequate support for his/her specific needs, in each particular daily environment, in order to be successful and participate along with peers in everyday activities. According to the ICF framework, individualized additional supports may be required for any child who experience participation restrictions, regardless of the presence of a diagnosis (Hollenweger, 2017). Teachers and other professionals in educational settings would benefit from getting feedback from the present results as they are responsible for assessing children's needs and strengths, as well as environmental barriers and facilitators, in order to mobilize and provide adequate support, when needed, for ensure all children's right to fully participate in educational contexts.

\section{ETHICS STATEMENT}

The Portuguese National Data Protection Authority and the Committee for Monitoring Studies in Education Settings of the General Direction of the Ministry of Education approved all measures and data collection procedures (authorization no. $16785 / 2015$ and authorization no. 0535000001, respectively); informed consent was obtained from the preschool directors, preschool teachers and families. No additional ethical authorizations were required. The mentioned approvals analyzed all ethical issues regarding the study. Written informed consent was obtained from the preschool directors, preschool teachers and families.

\section{AUTHOR CONTRIBUTIONS}

Both VC and AP made substantial contributions: to the conception and design of the work; to the acquisition, analysis, and interpretation of data for the work; in the drafting process of the work and the revising process; to the final approval of the version to be published; and in ensuring that questions related to the accuracy or integrity of any part of the work were appropriately investigated and resolved.

\section{ACKNOWLEDGMENTS}

The authors would like to thank all teachers, directors, families, and children who participated in the study, as well as to all researchers collecting data during the project.

\section{FUNDING}

This paper is financed by FEDER funds through the Operational Competitiveness Program - COMPETE and by national funds through FCT - Portuguese Foundation for Science and Technology under the reference SFRH/BD/111211/2015; Centre of Psychology of Porto University, UID/PSI/000050/2013. 


\section{REFERENCES}

Abreu-Lima, I. P., Leal, T. B., Cadima, J., and Gamelas, A. M. (2013). Predicting child outcomes from preschool quality in Portugal. Eur. J. Psychol. Educ. 28, 399-420. doi:10.1007/s10212-012-0120-y

Adolfsson, M., Granlund, M., and Pless, M. (2012). Professionals' views of children's everyday life situations and the relation to participation. Disabil. Rehabil. 34, 7. doi:10.3109/09638288.2011.613519

Aguiar, C., Moiteiro, A. R., and Pimentel, J. S. (2010). Classroom quality and social acceptance of preschoolers with disabilities. Infants Young Child. 23, 1. doi:10.1097/IYC.0b013e3181c9766e

Almqvist, L. (2006). Patterns of engagement in young children with and without developmental delay. J. Policy Pract. Intell. Disabil. 3, 1. doi:10.1111/j. 1741-1130.2006.00054.x

Aydoğan, C. (2012). Influences of Instructional and Emotional Classroom Environments and Learning Engagement on Low-Income Children's Achievement in the Prekindergarten Year. Dissertation/PhD's thesis, Faculty of the Graduate School of Vanderbilt University, Vanderbilt, USA.

Aydoğan, C., Farran, D. C., and Sağsöz, G. (2015). The relationship between kindergarten classroom environment and children's engagement. Eur. Early Child. Educ. Res. J. 23, 5. doi:10.1080/1350293X.2015.1104036

Bagnato, S. J. (2007). Authentic Assessment for Early Childhood Intervention: Best Practices. New York, NY: Guilford Press.

Bagnato, S. J., Niesworth, J. T., and Pretti-Frontczak, K. (2010). LINKing Authentic Assessment and Early Childhood Intervention: Best Measures for Best Practices, 2nd Edn. Baltimore, MD: Paul H. Brookes.

Bailey, D. B., and Wolery, M. (1992). Teaching Infants and Preschoolers with Disabilities, 2nd Edn. Columbus, OH: Macmillan.

Blasco, P. M., Bailey, D. B., and Burchinal, M. A. (1993). Dimensions of mastery in same-age and mixed-age integrated classrooms. Early Child. Res. Q. 8, 193-206. doi:10.1016/S0885-2006(05)80090-0

Brillante, P. (2017). The Essentials: Supporting Young Children with Disabilities in the Classroom. Washington: NAEYC.

Bronfenbrenner, U. (1979). The Ecology of Human Development. Cambridge, MA: Harvard University Press.

Bronfenbrenner, U., and Morris, P. A. (2007). "The bioecological model of human development," in Handbook of Child Psychology, 6th Edn, Vol. 1, eds W. Damon and R. M. Lerner (Hoboken, NJ: Wiley), 793-828.

Brown, M., and Bergen, D. (2002). Play and social interaction of children with disabilities at learning/activity centers in an inclusive preschool. J. Res. Child. Educ. 17, 1. doi:10.1080/02568540209594996

Caetano, A. (2014). Caraterização de crianças em situação de risco no centro histórico do Porto [Characterization of At-risk Children in the Historical Center of Porto]. Dissertation/Master dissertation, Faculty of Psychology and Educational Sciences of Porto University, Porto, Portugal.

Campbell, W. N., and Skarakis-Doyle, E. (2007). School-aged children with SLI: the ICF as a framework for collaborative service delivery. J. Commun. Disord. 40, 6. doi:10.1016/j.jcomdis.2007.01.001

Casey, A. M., McWilliam, R. A., and Sims, J. (2012). Contributions of incidental teaching, developmental quotient, and peer interactions to child engagement. Infants Young Child. 25, 2. doi:10.1097/IYC.0b013e31824cbac4

Castro, S. (2012). The Assessment-Intervention Process of Young Children with Autism: Contributions of the International Classification of Functioning, Disability and Health for Children and Youth. Dissertation/PhD thesis, Faculty of Psychology and Educational Sciences of Porto University, Porto, Portugal.

Castro, S., Pinto, A., and Simeonsson, R. J. (2014). Content analysis of Portuguese individualized education programmes for young children with autism using the ICF-CY framework. Eur. Early Child. Educ. Res. J. 22, 1. doi:10.1080/1350 293X.2012.704303

Castro, S., and Pinto, A. I. (2013). Identification of core functioning features for assessment and intervention in autism spectrum disorders. Disabil. Rehabil. 35, 125-133. doi:10.3109/09638288.2012.690494

Castro, S., and Pinto, A. I. (2015). Matrix for assessment of activities and participation: measuring functioning beyond diagnosis in young children with disabilities. Dev. Neurorehabil. 18, 3. doi:10.3109/17518423.2013.806963

Castro, S., and Pinto, A.I. (2012). Matrix of Assessment of Activities and Participation. [Unpublished manual], Faculty of Psychology and Educational Sciences of Porto University, Porto, Portugal.
Cohen, J. (1988). Statistical Power Analysis for the Behavioral Sciences, 2nd Edn. Hillsdale, NJ: Lawrence Erlbaum Associates.

Coster, W., and Khetani, M. A. (2008). Measuring participation of children with disabilities: issues and challenges. Disabil. Rehabil. 30, 8. doi:10.1080/ 09638280701400375

Coster, W., Law, M., Bedell, G., Khetani, M., Cousins, M., and Teplicky, R. (2012). Development of the participation and environment measure for children and youth: conceptual basis. Disabil. Rehabil. 34, 3. doi:10.3109/09638288.2011. 603017

Craig-Unkefer, L. A., and Kaiser, A. P. (2002). Improving the social communication skills of at-risk preschool children in a play context. Top. Early Child. Spec. Educ. 22, 3-13. doi:10.1177/027112140202200101

Cryer, D., Tietze, W., Burchinal, M., Leal, T., and Palacios, J. (1999). Predicting process quality from structural quality in preschool programs: a cross-country comparison. Early Child. Res. Q. 14, 339-361. doi:10.1016/S0885-2006(99)00017-4

de Kruif, R. E. L., and McWilliam, R. A. (1999). Multivariate relationships among development age, global engagement and observed child engagement. Early Child. Res. Q. 14, 515-536. doi:10.1016/S0885-2006(99)00028-9

de Sam Lazaro, S. L. (2017). The importance of authentic assessments in eligibility determination for infants and toddlers. J. Early Intervention 39, 2. doi:10.1177/1053815116689061

Decree-Law 3/2008. (2008). Decreto-Lei no.o 3/2008 de 7 de Janeiro [Decree-Law No.3/2008 of January 7]. Diário da República, 1. série, No.o 4. Ministério da Educação.

Downer, J. T., Rimm-Kaufman, S. E., and Pianta, R. C. (2007). How do classroom conditions and children's risk for school problems contribute to children's behavioral engagement in learning? School Psych. Rev. 36, 3. doi:10.1080/ 10409289.2016.1175240

Dunst, C. D., Bruder, M. B., Trivette, C. M., and Hamby, D. W. (2006). Everyday activity settings, natural learning environments, and early intervention practices. J. Policy Pract. Intell. Disabil. 3, 1. doi:10.1111/j.1741-1130.2006.00047.x

Ellingsen, K. M. (2011). Deriving Developmental Code Sets from the International Classification of Functioning, Disability and Health - for Children and Youth (ICF-CY). Dissertation/PhD thesis, University of North Carolina at Chapel Hill, Chapel Hill.

Eriksson, L., and Granlund, M. (2004). Conceptions of participation in students with disabilities and persons in their close environment. J. Dev. Phys. Disabil. 16, 9-245. doi:10.1023/B:JODD.0000032299.31588.fd

Eriksson, L., Welander, J., and Granlund, M. (2007). Participation in everyday school activities for children with and without disabilities. J. Dev. Phys. Disabil. 19, 5. doi:10.1007/s10882-007-9065-5

Farran, D., and Anthony, K. (2014). Child Observation in Preschool. Nashville, TN: Peabody Research Institute, Vanderbilt University. [Unpublished manual].

Ferreira, T., Coelho, V., and Pinto, A. I. (2012). "Early childhood intervention practices based on evidence: the case of participation," in European Conference on Developmental Psychology ECDP, ed. Medimond International Proceedings (Bolonha, Italy: CD-ROM), 485-490.

Fuhs, M. W., Farran, D. C., and Nesbitt, K. T. (2013). Preschool classroom processes as predictors of children's cognitive self-regulation skills development. Sch. Psychol. Q. 28, 347-359. doi:10.1037/spq0000031

Gamelas, A. M. (2003). Contributos para o Estudo da Ecologia de Contextos PréEscolares Inclusivos [Contributions to the Study of Inclusive Preschool Contexts Ecology]. Lisbon: Ministry of Education.

Grande, C. (2013). Estudo do impacto das interacções educadora-criança no envolvimento das crianças com necessidades educativas especiais em contexto de creche e de jardim-de-infância [Study of the Impact of Teacher-Child Interactions in the Engagement of Children with Special Educational Needs in the Context of Day Care and Preschool]. Lisbon: Calouste Gulbenkian Foundation.

Granlund, M. (2013). Participation - challenges in conceptualization, measurement and intervention. Child Health Care Dev. 39, 4. doi:10.1111/cch.12080

Grisham-Brown, J., and Pretti-Frontczak, K. (2011). Assessing Young Children in Inclusive Settings: The Blended Practices Approach. Baltimore: Brookes Publishing Company.

Guichard, S., and Grande, C. (2017). The role of environment in explaining frequency of participation of pre-school children in home and community activities. Int. J. Dev. Disabil. doi:10.1080/20473869.2017.1378160

Guralnick, M. (2008). International perspectives on early intervention: a search for common ground. J. Early Intervention 30, 2. doi:10.1177/1053815107313483 
Guralnick, M. J., Hammond, M. A., Connor, R. T., and Neville, B. (2006). Stability, change, and correlates of the peer relationships of young children with mild developmental delays. Child Dev. 77, 2. doi:10.1111/j.1467-8624. 2006.00872.x

Hollenweger, J. (2017). “Applying ICF in education and care," in An Emerging Approach for Education and Care: Implementing a Worldwide Classification of Functioning and Disability, eds S. Castro and O. Palikara (London/New York: Routledge), 23-36.

Imms, C., and Granlund, M. (2014). Participation: are we there yet. Austr. Occup. Ther. J. 61, 5. doi:10.1111/1440-1630.12166

Imms, C., Granlund, M., Wilson, P. H., Steenbergen, B., Rosenbaum, P. L., and Gordon, A. M. (2017). Participation, both a means and an end: a conceptual analysis of processes and outcomes in childhood disability. Dev. Med. Child Neurol. 59, 1. doi:10.1111/dmcn.13237

Ivory, J. J., and McCollum, J. A. (1999). Effects of social and isolate toys on social play in an inclusive setting. J. Spec. Educ. 32, 238-243. doi:10.1177/ 002246699903200404

Jamison, K. R., Forston, L. D., and Stanton-Chapman, T. L. (2012). Encouraging social skill development through play in early childhood special education classrooms. Young Except. Child. 15, 2. doi:10.1177/1096250611435422

Kontos, S., and Keyes, L. (1999). An ecobehavioral analysis of early childhood classrooms. Early Child. Res. Q. 14, 35-50. doi:10.1016/S0885-2006(99)80003-9

Laevers, F. (1997). Assessing the quality of childcare provision: "involvement" as criterion. Res. Early Child. 3, 151-165.

Laevers, F. (2006). Making Care and Education More Effective through Wellbeing and Involvement: An Introduction to Experiential Education. Working Together: Making a Difference for South Australian Children: Our Children the Future Early Childhood Seminar, 8 November. South Australia: Department of Education and Children's Services.

Lai, D., Tseng, Y., Hou, Y., and Guo, H. R. (2012). Gender and geographic differences in the prevalence of autism spectrum disorders in children: analysis of data from the national disability registry of Taiwan. Res. Dev. Disabil. 33, 3. doi:10.1016/j.ridd.2011.12.015

Lebeer, J., Birta-Szekely, N., Demeter, K., Bohacs, K., Candeias, A. A., Sonnesyn, G., et al. (2012). Re-assessing the current assessment practice of children with special education needs in Europe. Sch. Psychol. Int. 13, 1. doi:10.1177/0143034311409975

Lillvist, A. (2010). Observations of social competence of children in need of special support based on traditional disability categories versus a functional approach. Early Child Dev. Care 180, 9. doi:10.1080/03004430902830297

Luthar, S. S., Crossman, E. J., and Small, P. J. (2015). "Resilience and adversity," in Handbook of Child Psychology and Developmental Science, eds R. M. Lerner and M. E. Lamb (New York: Wiley), 247-286.

Luttropp, A., and Granlund, M. (2010). Interaction - it depends - a comparative study of interaction in preschools between children with intellectual disability and children with typical development. Scand. J. Disabil. Res. 12, 3. doi:10.1080/15017410903175677

Macy, M. G., Bagnato, S. J., Macy, R. S., and Salaway, J. (2015). Conventional tests and testing for early intervention eligibility: is there an evidence base? Infants Young Child. 28, 182-204. doi:10.1097/IYC.0000000000000032

Majnemer, A. (2012). Measures for Children with Developmental Disabilities: An ICF-CY Approach. London: Mac Keith Press.

Malone, D. M., Stoneman, Z., and Langone, J. (1994). Contextual variation of correspondences among measures of play and developmental level of preschool children. J. Early Intervention 18, 199-215. doi:10.1177/105381519401800207

McConnell, S. R., Wackerle-Hollman, A. K., Roloff, T. A., and Rodriguez, M. (2014). Designing a measurement framework for response to intervention in early childhood programs. J. Early Intervention 36, 4. doi:10.1177/1053815115578559

McManus, B. M., Carle, A. C., and Rapport, M. J. (2014). Classifying infants and toddlers with developmental vulnerability: who is most likely to receive early intervention? Child. Care Health Dev. 40, 2. doi:10.1111/cch.12013

McWilliam, R. A. (2005). Foundations for Learning in a Modern Society. Nashville, TN: Center for Child Development, Vanderbilt University Medical Center. [Unpublished Manuscript].

McWilliam, R. A., and Bailey, D. B. (1992). "Promoting engagement and mastery," in Teaching Infants and Preschoolers with Disabilities, eds D. B. Bailey and M. Wolery (New York: MacMillan Publishing Company), 230-255.
Murphy, R. (2006). "Evaluating new priorities in assessment in higher education," in Innovative Assessment in Higher Education, eds C. Bryan and K. Clegg (London: Routledge), 37-46.

Nesbitt, K. T., Farran, D. C., and Fuhs, M. W. (2015). Executive function skills and academic achievement gains in prekindergarten: contributions of learningrelated behaviors. Dev. Psychol. 51, 7. doi:10.1037/dev0000021

Norwich, B. (2016). Conceptualizing special educational needs using a biopsychosocial model in England: the prospects and challenges of using the International Classification of Functioning framework. Front. Educ. 1:5. doi:10.3389/ feduc.2016.00005

Odom, S. L., and Bailey, D. B. (2001). "Inclusive preschool programs: classroom ecology and child outcomes," in Early Childhood Inclusion: Focus on Change, ed. M. J. Guralnick (Baltimore: Brooks), 253-276.

Odom, S. L., McConnell, S. R., and McEvoy, M. A. (1992). "Peer-related social competence and its significance for young children with disabilities: issues and strategies for intervention," in Social Competence of Young Children with Disabilities: Issues and Strategies for Intervention, eds S. L. Odom, S. R. McConnell, and M. A. McEvoy (Baltimore, MD: Brookes), 3-35.

Pinto, A., Grande, C., Abreu-lima, I., Cadima, J., Mata, L., Pimentel, J., et al. (2014). Estudo de avaliação das Orientações Curriculares e da qualidade na educação pré-escolar: Relatório final dos Estudos de Caso [Study of the Assessment of the Curriculum Guidelines and Quality in Preschool: Final Report of the Case Studies]. Portugal: Ministry of Education. [unpublished manuscript].

Pinto, A. I., Barros, S., Aguiar, C., Pessanha, M., and Bairrão, J. (2006). Relações entre idade desenvolvimental, dimensões do comportamento adaptativo e envolvimento observado [Relationships between developmental age, dimensions of adaptive behavior and observed engagement]. Análise Psicológica. 24, 4. doi:10.14417/ap.538

Pinto, A. I., Grande, C., Aguiar, C., de Almeida, I. C., Felgueiras, I., Pimentel, J. S., et al. (2012). Early childhood intervention in Portugal: an overview based on the developmental systems model. Infants Young Child. 25, 4. doi:10.1097/ IYC.0b013e3182673e2b

Raspa, M. J., McWilliam, R. A., and Ridley, S. M. (2001). Child care quality and children's engagement. Early Educ. Dev. 12, 2. doi:10.1207/s15566935eed1202_3

Roper, N., and Dunst, C. J. (2003). Communication intervention in natural learning environments: guidelines for practice. Infants Young Child. 16, 215-226. doi:10.1097/00001163-200307000-00004

Rubin, K. H., Maioni, T. L., and Hornung, M. (1976). Free play behaviors in middle- and lower-class preschoolers: Parten and Piaget Revisited. Child Dev. 47, 2. doi:10.1111/1467-8624.ep12189769

Sanches-Ferreira, M., Simeonsson, R. J., Silveira-Maia, M., and Alves, S. (2015). Evaluating implementation of the International Classification of Functioning, Disability and Health in Portugal's special education law. Int. J. Inclusive Educ. 19, 5. doi:10.1080/13603116.2014.940067

Semrud-Clikeman, M. (2007). Social Competence in Children. US: Springer.

Sigafoos, J., Roberts-Pennell, D., and Graves, D. (1999). Longitudinal assessment of play and adaptive behavior in young children with developmental disabilities. Res. Dev. Disabil. 20, 147-161. doi:10.1016/S0891-4222(98)00038-9

Silva, I. L., Marques, L., Mata, L., and Rosa, M. (2016). Orientações Curriculares para a Educação Pré-Escolar [Curriculum Guidelines for Preschool Education]. Portugal: Ministry of Education and General Direction of Education.

Silveira-Maia, M., Lopes-dos-Santos, P., and Sanches-Ferreira, M. (2017). How the use of the international classification of functioning, disability and health for children and youth changed the individualized education programs in Portugal. Int. J. Inclusive Educ. 21, 5. doi:10.1080/13603116.2016. 1218950

Simeonsson, R. J., Carlson, D., Huntington, G. S., McMillen, J. S., and Brent, J. L. (2001). Students with disabilities: a national survey of participation in school activities. Disabil. Rehabil. 23, 2. doi:10.1080/096382801750058134

Simeonsson, R. J., Leonardi, M., Lollar, D., Bjorck-Akesson, E., Hollenweger, J., and Martinuzzi, A. (2003). Applying the international classification of functioning, disability and health (ICF) to measure childhood disability. Disabil. Rehabil. 25, 11-12. doi:10.1080/09638280310000

Sjöman, M., Granlund, M., and Almqvist, L. (2016). Interaction processes as a mediating factor between children's externalized behaviour difficulties and engagement in preschool. Early Child Dev. Care 186, 10. doi:10.1080/030044 30.2015 .1121251 
Turnbull, J., and Jenvey, V. B. (2006). Criteria used by adults and children to categorize subtypes of play. Early Child Dev. Care 176, 5. doi:10.1080/ 03004430500258172

UN General Assembly. (2007). Convention on the Rights of Persons with Disabilities: Resolution/Adopted by the General Assembly, A/RES/61/106. Available at: http:// www.refworld.org/docid/45f973632.html

UNESCO. (1994). The UNESCO Salamanca Statement and Framework for Action on Special Needs Education. Paris: UNESCO.

Wolery, M., Paucca, T., Brashers, M. S., and Grant, S. (2000). Quality of Inclusive Experiences Measure. Chapel Hill: University of North Carolina, FPG Child Development Center.

World Health Organization. (2001). International Classification of Functioning, Disability and Health (ICF). Geneva: WHO.

World Health Organization. (2007). International Classification of Functioning, Disability and Health-Version for Children and Youth (ICF-CY). Geneva: WHO.
World Health Organization. (2013). How to Use the ICF: A Practical Manual for Using the International Classification of Functioning, Disability and Health (ICF). Geneva: WHO.

Xu, Y. (2010). Children's social play sequence: parten's classic theory revisited. Early Child Dev. Care 180, 4. doi:10.1080/03004430802090430

Conflict of Interest Statement: The authors declare that the research was conducted in the absence of any commercial or financial relationships that could constitute a potential conflict of interest.

Copyright (C) 2018 Coelho and Pinto. This is an open-access article distributed under the terms of the Creative Commons Attribution License (CC BY). The use, distribution or reproduction in other forums is permitted, provided the original author(s) and the copyright owner are credited and that the original publication in this journal is cited, in accordance with accepted academic practice. No use, distribution or reproduction is permitted which does not comply with these terms. 